\title{
Age-related differences in corrected and inhibited pointing movements
}

\author{
Stéphanie Rossit • Monika Harvey
}

Received: 12 June 2007 / Accepted: 30 August 2007 / Published online: 25 September 2007

(C) Springer-Verlag 2007

\begin{abstract}
It has been widely reported that aging is accompanied by a decline in motor skill performance and in particular, it has been shown that older subjects take longer to adapt their ongoing reach in response to a target location shift. In the present experiment, we investigated the influence of aging on the ability to perform trajectory corrections in response to a target jump, but also assessed inhibition by asking a younger and an older group of participants to either adapt or stop their ongoing movement in response to a target location change. Results showed that although older subjects took longer to initiate, execute, correct and inhibit an ongoing reach, they performed both tasks with the same level of accuracy as the younger sample. Moreover, the slowing was also observed when older subjects were asked to point to stationary targets. Our findings thus indicate that aging does not specifically influence the ability to perform or inhibit fast online corrections to target location changes, but rather produces a general slowing and increased variability of movement planning, initiation and execution to both perturbed and stationary targets. For the first time, we demonstrate that aging is not accompanied by a decrease in the inhibition of motor control.
\end{abstract}

Keywords Aging - Double-step paradigm . Online corrections · Inhibitory motor control

S. Rossit · M. Harvey $(\square)$

Department of Psychology, University of Glasgow, Glasgow G12 8QB, Scotland, UK

e-mail: m.harvey@psy.gla.ac.uk

\section{Introduction}

The double-step paradigm has been widely used to investigate the online control of goal directed movements and has repeatedly shown that in young adults a target location change at the outset of a pointing movement elicits a fast online correction to accurately reach the target (Goodale et al. 1986; Paulignan et al. 1991; Prablanc and Martin 1992). The delay of these corrections is believed to be 100 $150 \mathrm{~ms}$ and the change in movement trajectory has been shown to be smooth and without abrupt changes to the velocity profiles and movement times (Goodale et al. 1986; Paulignan et al. 1991; Prablanc and Martin 1992). Such findings have led to the conclusion that an ongoing pointing movement to a visual target can be corrected without the reprogramming of a new motor output.

Interestingly, these rapid online corrections in response to target location changes have been found not to require awareness of the location shift (Pélisson et al. 1986; Castiello et al. 1991; Johnson et al. 2002) and under certain conditions participants are even unaware of their own limb modifications (Chua and Enns 2005; Day and Lyon 2000; Fecteau et al. 2001; Prablanc and Martin 1992; Goodale et al. 1986; Pélisson et al. 1986). Recently, it has also been shown that such corrections are so fast that they can prevail in spite of an instructed stopping response (Day and Lyon 2000; Pisella et al. 2000). For example, Day and Lyon (2000) investigated the ability to control online corrections by asking healthy subjects to either move in the same or opposite direction of the target shift. They found that, in response to the location shift, only $24 \%$ of such corrections could be suppressed by the intention to move away from the target. As these rapid corrections seem almost irreversible they are seen as evidence for a strong 'automatic pilot' of the hand, believed to be mediated by neural structures of 
the visual dorsal stream (posterior parietal cortex; Goodale and Milner 2005; Pisella et al. 2000; Gréa et al. 2002).

It is widely documented that motor skills decline with age compared to young adults, older subjects present a slowing of motor responses and their execution, a higher variability in reaction and movement time (Salthouse 2000; Yan et al. 1998; Yan 2000; Welford et al. 1969; Pratt et al. 1994; Walker et al. 1997) and a reduced smoothness of the movement (Yan 2000). Surprisingly though, just how aging affects the kinematics of automatic goal-directed movements has not received a great degree of attention in the literature, although it has been tentatively suggested that older adults have more difficulty achieving automaticity. For example, Wu and Hallet (2005) asked older adults to perform different sequential (and previously learned) fingertapping movements simultaneously to a secondary lettercounting task. They found that, although the older subjects took longer to achieve this automaticity than the younger group, they achieved it to the same level of accuracy. Moreover, functional magnetic resonance imaging revealed that the older subjects required greater brain network activity than the younger adults to perform the movements automatically. The authors suggest that this reliance on greater networks may be the main reason as to why older subjects have more difficulty in achieving automaticity. In line with this hypothesis, it has been shown that elderly subjects exhibit altered brain activation patterns during simple isolated finger movements (Calautti et al. 2001; Hutchison et al. 2002; Mattay et al. 2002), hand movements (Hutchison et al. 2002; Ward and Frackowiak 2003) and cyclical hand and/or foot movements (Heuninckx et al. 2005).

To our knowledge the only study that specifically investigated age-related differences using the double-step paradigm in relation to reaching, is the one by Sarlegna (2006) assessing how elderly participants amend their hand movements towards a displaced target. Six young and six older participants were asked to direct a pointer at either continuously visible, flashed or non-visible targets that could either stay stationary or unpredictably jump to the left or right of the central target at movement onset. It was found that older subjects took significantly longer to perform a corrective movement towards a displaced target. Based on this finding the authors argued that with aging comes a temporary impairment of online visual feedback control processes, accomplished by the posterior parietal cortex. However, end-point accuracy parameters were not assessed in this study. This would have been helpful as it has been previously suggested that older subjects try to compensate for slower processing speed by slowing their responses in order to be accurate, a hypothesis known as 'speed/accuracy trade-off' (e.g. Salthouse 1979). The present study was aimed at clarifying this point.
More importantly, we compared the performance between older and younger participants in both a location go and a location stop condition using the same protocol as Pisella et al. (2000). Detailed kinematic analysis of pointing movements in the location go condition allowed us to examine if there was indeed a decreased ability to perform movements to perturbed targets with aging. Additionally, location stop trials can shed further light on the automaticity of corrective responses as we examined whether participants, in the stop condition, initiated any trajectory adjustments immediately prior to the interruption of their movement.

Finally, and more importantly, with the stop condition, we examined if aging exerts any influence on the ability to intentionally interrupt a movement in-flight. To our knowledge this examination has not been done.

In fact, although brain structures responsible for goaldirected movements have been studied extensively in the last century, underlying neural mechanisms regarding the voluntary control of ongoing movements have just recently become a matter of interest: using single-unit recordings, Schall and colleagues (Schall 2001; Schall et al. 2000; Schall et al. 2002) have shown a number of frontal cortical areas involved in this process, with complimentary evidence coming from fMRI testing in humans (Aron and Poldrack 2006; Curtis et al. 2004).

Moreover, in a behavioural study, Mirabella et al. (2006) tested the ability of young subjects to perform either fast reaches toward a visual target in the same or opposite side of the reaching arm or, in stop trials, to withhold a command movement whenever an unpredictable stop signal was presented. They found that as the time gap between the go and stop signals increased, subjects increasingly failed to inhibit the motor response and that the stop time reaction time for reaching movements was approximately $200 \mathrm{~ms}$. In addition, it was observed that subjects were faster when stopping their movement towards visual targets appearing in the same side of the reaching arm, which they interpreted as evidence for independent processes for stop and go responses that share a common mechanism when under the control of the same hemisphere.

This paradigm has not yet been applied to elderly subjects, but Potter and Grealy (2006) assessed the ability of elderly adults to inhibit a primed movement plan in favour of a novel one and found that the majority of adults failed to inhibit responses by the time they reached their sixties. This finding was extended in a very recent study (Potter and Grealy 2007) using a go/no-go task, again showing that older subjects produced more inhibition failures. Kramer et al (1994) also found that elderly compared to younger adults, were less able to inhibit overt responses: when both elderly and young adults were asked to perform a series of tasks measuring inhibitory functions, it was found that older adults had more difficulty than younger ones in stopping 
the overt response and adopting new rules, indicating that aging decreases the ability to inhibit an overt response in favour of an intentional response. In line with this claim, Olincy et al. (1997) found age-related changes in an antisaccade task, in that the proportion of misfixations to the onset stimulus (rather than away from it) increased linearly with age. Moreover, older adults have greater difficulty in intentionally suppressing attentional allocation to onset distracters (Pratt and Bellomo 1999) and are more susceptible to attentional capture by transient events in the periphery of the visual field (Lincort et al. 1997). It is surprising then that Kramer et al. $(1999,2000)$ found equivalent patterns of oculomotor capture for old and young adults in that both groups misdirected their eyes to the task irrelevant onsets on an equivalent number of trials. Colcombe et al. (2003) further extended these findings of age equivalence from onset to colour singleton distracters.

Therefore, despite the wide-ranging research regarding the relationship between inhibitory functions and agerelated cognitive decline, just how these functions influence the control of action has not been investigated comprehensively and it is indeed hard to predict the behaviour of the elderly subjects. Based on the studies reviewed here we would expect elderly subjects to be delayed in the onset of their online correction to a target perturbation (see Sarlegna 2006), but possibly be accurate in carrying out such a correction. Regarding the movement inhibition in the stop condition, we would expect impairment with age, yet the oculomotor capture results by Kramer et al. (1999; 2000) would not support such an assumption.

\section{Method}

\section{Participants}

Eight older subjects (four males and four females, mean age 72.88, SD 3.98) and eight younger controls (four males and four females, mean age 20.88, SD 0.35) were recruited and participated voluntarily in this experiment. All participants were right-handed according to the Annett Handedness Inventory (Annett 1967), had normal or corrected-tonormal visual acuity, were healthy with no past history of neurological disease and did not suffer from confusion, general mental deterioration or psychiatric disorders. Ethical approval was granted by the local ethics committee and all subjects gave their informed consent prior to participation in the study.

\section{Apparatus}

Targets were white circles (7 $\mathrm{mm}$ of diameter) projected (HITACHI CP-X345 Multimedia LCD Projector, refresh rate of $60 \mathrm{~Hz}$ ) onto a horizontal Perspex box $(77 \mathrm{~cm}$ broad / $97 \mathrm{~cm}$ length $/ 30 \mathrm{~cm}$ high) via a reflection mirror $(3 \mathrm{~mm}$ thick, $60 \times 60 \mathrm{~cm}$ ). The box was placed on top of a wooden table $(63 \mathrm{~cm}$ high, $99 \mathrm{~cm}$ long, $39 \mathrm{~cm}$ broad) at which the subjects were comfortably seated. Targets were visible only when illuminated and no tactile information about their positions was available. They were located at $-40 \mathrm{~mm}$ (left hemispace) and $+40 \mathrm{~mm}$ (right hemispace) with respect to the central target $(0 \mathrm{~mm})$. The central target was located $400 \mathrm{~mm}$ in front of the start trigger, aligned with the centre of the box. At the start of each trial, the right index finger rested on the start trigger, aligned to the subject's sagittal midline.

\section{Design and procedure}

We used a location go versus location stop paradigm. In the location go condition (also called double-step) subjects had to point to a target, which could unexpectedly jump to the right or left from the central position. In this condition, participants were instructed to point to the target and if it jumped to follow it to its new location. In the location stop condition, subjects were instructed to stop their movement in response to the target location shift and return to the start position. For both conditions participants were instructed to perform their movements as quickly and as accurately as possible with their right index finger.

To preclude any predictive behaviour, targets were unperturbed in $70 \%$ of the trials. In $30 \%$ of the trials the target changed its position (perturbation trials). This shift was triggered by the release of the start button. In half of the perturbation trials, the target shifted to the right, in the other half it shifted to the left. The two conditions were given in separate blocks and block order was counterbalanced across participants. Each block contained 18 practise trials ( 6 for each target final position) and 200 experimental trials.

After pressing the start trigger $(1,000 \mathrm{~ms})$ the target was illuminated and a tone $(800 \mathrm{~Hz})$ cued subjects to perform the movement. One second after the start trigger release a pacing tone came on, announcing the end of the trial. Target positions remained visible until the end of the trial and were presented in a randomised order. Calibration coordinates were obtained at the end of each session, by continuous illumination of each target, one by one, allowing the subjects to adjust their terminal fingertip position until they felt that they had perfectly occluded the target. There were three calibration trials per target and three for the start position.

Pointing responses were recorded by sampling the position of a magnetic marker, attached to the tip of the index finger, at a rate of $108 \mathrm{~Hz}$, using an electro-magnetic motion analysis system (Minibird, Ascension Technology Inc.). The start trigger, the online recordings and the stimuli 
presentation were simultaneously controlled and timed by a PC, by means of a Virtual Instrument generated with LabView software (National Instruments).

\section{Data analysis}

Data obtained from the recordings was analysed offline. Start of the movement was defined by using a velocitybased criterion of $0 \mathrm{~mm} / \mathrm{s}$. For the location go condition, the end of the movement was determined by the kinematic data, using a velocity-based criterion of $50 \mathrm{~mm} / \mathrm{s}$.

A trial-by-trial analysis was performed to detect the time frame in which the $x$ position was altered in order to make a correction to the jump (correction time). To do so following Pisella et al. (2000), 95\% confidence intervals of the $x$ position were calculated for the non-perturbed trials. Perturbed trials were considered as corrected when the $x$ value was below or above the confidence interval at the end of the movement, allowing a classification of trials as either corrected or non-corrected. The end-point accuracy parameters were the constant angular error relative to the ideal reach (see also Cressman et al. 2006), calculated for each trial based on the calibration coordinates, as well as the variable error (i.e. the standard deviation of the constant angular error). The following dependent variables were analysed: proportion of successful corrections and for corrected trials only: correction time, movement time, peak velocity, constant angular error and variable error.

For the location stop condition, trials were classified as stop or non-stop using a Virtual Instrument that detected the first frame prior to the velocity reversal in the $y$-axis. The end of the movement was identified as the frame before the velocity in the $y$-axis went negative (pullback) provided that the velocity fell below $100 \mathrm{~mm} / \mathrm{s}$ within $50 \mathrm{~ms}$ of the first frame becoming negative. Trials meeting this criteria were classified as successful stops. We also used this criteria to obtain the time it took the subject to stop the movement before the pullback (stop time).

In addition, the same classification of corrected or noncorrected trials as in the location go condition was performed to identify whether participants made a correction towards the target location shift before pulling back. If yes, these types of responses were classified as disallowed corrections and measured in proportions. Therefore, for stop trials the following parameters were analysed: proportion of successful stops, stop time and proportion of disallowed corrections. Stop time was analysed for successful stop trials only.

Means for each of the participants were computed for each variable and proportion results were submitted to an arcsin transformation before statistical analysis. Post hoc comparisons were made with the Bonferroni method, $P<0.05$.

\section{Results}

Reaction time (RT)

To investigate the influence of aging on reaction time, we performed a mixed design ANOVA with age (older adults, younger adults) as a between factor and condition (location go, location stop) as a within factor. As expected older participants had significantly longer RTs $\left(F_{(1,14)}=5.01\right.$, $P<0.05$ ), but both age groups showed significantly longer RTs in the location stop compared to the location go condition $\left(F_{(1,14)}=7.63, P<0.05\right.$; see Table 1$)$. No significant interaction between age and condition was found.

\section{Location go condition}

Proportion of successful corrections and correction time were assessed with a mixed design ANOVA with age as a between and side (leftward or rightward perturbation) as the within factor (see Table 2).

For the proportion of successful corrections, there were no main effects of age nor side, but a significant interaction $\left(F_{(1,14)}=16.00, P=0.001\right)$. Pairwise comparisons showed that the older group made significantly fewer corrections in response to a left target jump and significantly more corrections to a rightward one $(P<0.05)$, while the opposite pattern was observed for the younger sample $(P<0.05)$.

Regarding correction time, we found that the older group took significantly longer to initiate their corrections than the younger sample $\left(F_{(1,14)}=16.98, P=0.001\right)$. Both groups were affected by side in that all participants corrected their movements significantly later when the perturbation occurred in a leftward direction $\left(F_{(1,14)}=33.79\right.$, $P<0.001)$. No interaction was found.

A mixed ANOVA with age as a between and trial type (leftward perturbation, rightward perturbation, non-perturbed) as a within factor was performed separately for each of the following variables: movement time, peak velocity, angular and variable error.

For movement time, as expected, there was a significant main effect of aging with the older group taking markedly longer to complete their movement $\left(F_{(1,14)}=15.90\right.$, $P<0.001)$. We also found an effect of trial type $\left(F_{(1,14)}=74.62, P<0.001\right)$ : as expected, pair-wise comparisons showed that movement times were shorter to unperturbed

Table 1 Reaction time means and standard deviations (in parenthesis) in ms per group and condition

\begin{tabular}{lll}
\hline Group & Location go & Location stop \\
\hline Young adults & $1221.4(56.5)$ & $1245.2(72.2)$ \\
Older adults & $1286.7(99.6)$ & $1349.1(91.7)$ \\
\hline
\end{tabular}


Table 2 Means and standard deviations (in parenthesis) for performance characteristics of the location go condition, separately per group and trial type (left, right and non-perturbed)

\begin{tabular}{|c|c|c|c|c|}
\hline Variable & Group & Right & Left & Non-perturbed \\
\hline \multirow[t]{2}{*}{ Proportion of corrections } & Young adults & $0.92(0.1)$ & $0.99(0.1)$ & \\
\hline & Older adults & $1.0(0.0)$ & $0.96(0.0)$ & \\
\hline \multirow[t]{2}{*}{ Correction time (ms) } & Young adults & $237.8(12.0)$ & $264.5(12.1)$ & \\
\hline & Older adults & $289.8(40.4)$ & $325.8(39.6)$ & \\
\hline \multirow[t]{2}{*}{ Movement time (ms) } & Young adults & $413.5(35.6)$ & $455.4(24.8)$ & $388.1(32.0)$ \\
\hline & Older adults & $561.2(102.8)$ & $592.8(93.4)$ & $519.2(90.5)$ \\
\hline \multirow[t]{2}{*}{ Peak velocity $(\mathrm{mm} / \mathrm{s})$} & Young adults & $1934.4(169.9)$ & $1944.1(192.0)$ & $1938.0(32.0)$ \\
\hline & Older adults & $1566.1(272.6)$ & $1531.7(255.2)$ & $1535.6(247.7)$ \\
\hline \multirow[t]{2}{*}{ Angular error (deg) } & Young adults & $-0.07(0.6)$ & $-0.04(0.5)$ & $-0.09(0.4)$ \\
\hline & Older adults & $-0.005(0.5)$ & $-0.14(0.4)$ & $0.03(0.4)$ \\
\hline \multirow[t]{2}{*}{ Variable error (deg) } & Young adults & $0.81(0.2)$ & $0.53(0.1)$ & $0.54(0.0)$ \\
\hline & Older adults & $0.69(0.3)$ & $0.52(0.1)$ & $0.48(0.0)$ \\
\hline
\end{tabular}

than perturbed trials $(P<0.001$ for the left target perturbation; $P=0.001$ for the right target perturbation). In addition, all subjects took substantially longer to complete their movements when the target was perturbed to the left side compared to the right side or non-perturbed trials $(P<0.001)$. No interaction between the factors was found.

Only age was found to affect peak velocity, with the older group performing more slowly $\left(F_{(1,14)}=12.25\right.$, $P<0.01$ ).

Interestingly, regarding constant angular error no effect of age or trial type, nor an interaction was found. Both groups ended their movement on target for both perturbed and non-perturbed trials. For variable error, an effect of trial type was found $\left(F_{(1,14)}=9.80, P<0.01\right)$. Pairwise comparisons revealed that the end-point was more variable for right compared to left target perturbations and unperturbed trials $(P<0.01)$.

Location stop condition

Proportion of successful stops, proportion of disallowed corrections and stop time were assessed with mixed design ANOVAs with age as between and side (leftward or rightward perturbation) as the within factor (see Table 3).

For the proportion of stops, no main effects of age or side nor an interaction between the two factors were observed. Both age groups were similarly able to stop their movements in response to the left and right target displacements.

However, despite the stop instruction both age groups performed disallowed corrections in the direction of the perturbation. Interestingly, a main effect of side was found for the proportion of disallowed corrections in the stop condition $\left(F_{(1,14)}=4.70, P<0.05\right)$, with all participants
Table 3 Means and standard deviations (in parenthesis) for performance characteristics in perturbed trials of the location stop condition per group and side of perturbation

\begin{tabular}{llll}
\hline Variable & Group & Right & Left \\
\hline Proportion of stops & Young adults & $0.97(0.0)$ & $0.96(0.0)$ \\
& Older adults & $0.92(0.1)$ & $0.92(0.2)$ \\
Proportion of & Young adults & $0.62(0.2)$ & $0.48(0.2)$ \\
$\quad$ disallowed corrections & Older adults & $0.57(0.3)$ & $0.48(0.2)$ \\
Stop time (ms) & Young adults & $325.2(51.5)$ & $342.9(59.3)$ \\
& Older adults & $417.3(97.7)$ & $424.9(96.3)$ \\
\hline
\end{tabular}

presenting a higher proportion of corrections (despite the stop instruction) in response to a right target displacement, when compared to the left one. No effects of age nor an interaction were obtained for this variable.

Regarding stop time, similarly to correction time, the older group took significantly longer than the younger subjects to stop their movements $\left(F_{(1,14)}=4.96, P<0.05\right)$. There was also a main effect of side with participants taking longer to stop in response to a left rather than right target displacement $\left(F_{(1,14)}=5.26, P<0.05\right)$. No interaction was found.

\section{Movement trajectories}

In Fig. 1 we present the 2D vector means (in deg) by time slice per group per trial type (only successfully corrected and stopped trials were included). Vector trajectories were obtained by averaging trials per subject, separately for each final target position. To ensure that the graphs were homogeneous in terms of time slices across conditions and groups, we only included mean data points from 65 to 
Fig. 1 Mean 2D vector (deg) per time slice (ms) for the younger (a) and older (b) groups per final target position (left, right and non-perturbed trials) for the location go condition.

The traced line represents mean values of unperturbed trials with \pm 2 standard deviations

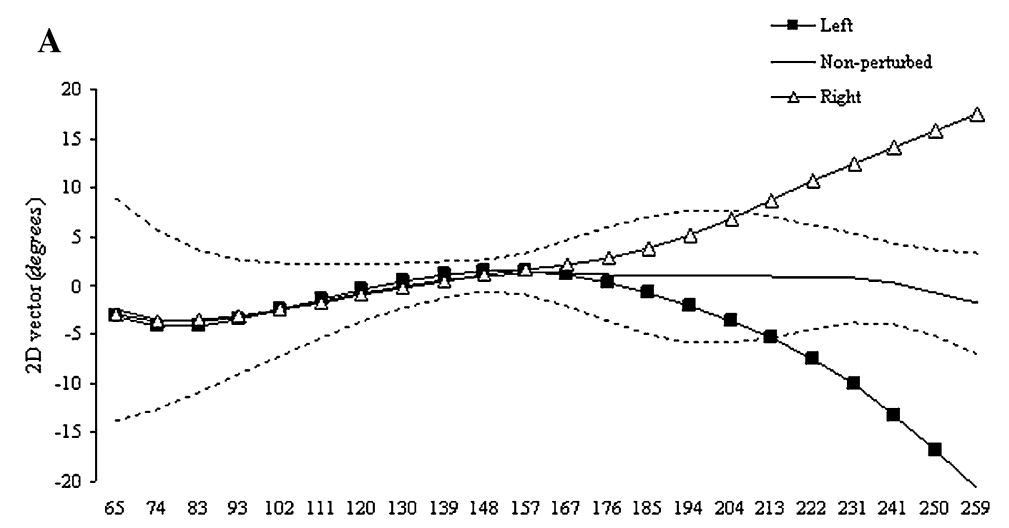

B

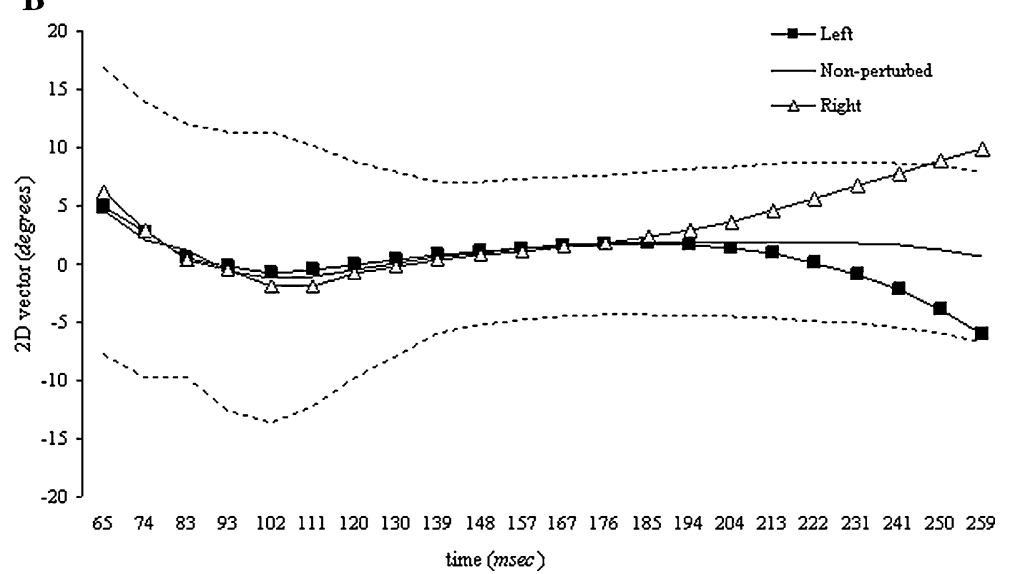

$259 \mathrm{~ms}^{1}{ }^{1}$ Graphs were plotted after calculating the overall $2 \mathrm{D}$ vector mean. We also included the lower and upper boundary of the unperturbed trials, by subtracting and summing its mean values with 2 standard deviations. This 2D vector trajectory mean with its two standard deviation bandwidth, allowed us to assess the correction and stop movement trajectories qualitatively.

Although this trajectory analysis is in agreement with the 95\% confidence intervals results it is a very conservative estimate. Therefore (as suggested by one of the referees), to obtain an earlier estimate of correction time, a paired $t$ test of the $2 \mathrm{D}$ vector coordinates was performed. Comparisons were made between unperturbed and left or right perturbed trials for each time slice for each group.

For the location go trials, both groups initiated their corrections in the direction of the perturbation to the left later than to the right target displacement, which confirms the effect of side obtained for correction time (see Fig. 1). The younger group (Fig. 1a) started correcting towards the rightward perturbation at around $204 \mathrm{~ms}$ and only at

\footnotetext{
${ }^{1}$ These time frames were selected as they contained $100 \%$ data points across all trials, conditions and subjects. Note that the graphs miss $56 \%$ of data points in the go condition and 55\% in the stop condition both at the beginning and end of the movement.
}

$213 \mathrm{~ms}$ towards the left perturbation. However, disregarding the strict criteria of the unperturbed trials bandwidth, the paired $t$ test showed that the trajectory started its deviation from the unperturbed mean trajectory as soon as $176 \mathrm{~ms}$ to the right and left $\left(t_{(7)}=-4.50, P<0.01\right.$; $t_{(7)}=3.71, P<0.01$, respectively).

The first notable characteristic of the older group's trajectory (Fig. 1b) is the higher variability of its bandwidth compared to that of the younger group. However, it seems that the trajectory is similar between groups, despite the slower deviation towards the perturbation and the higher variability in the older sample. Older participants started to correct their reaching at around $259 \mathrm{~ms}$ to the right and later to the left. Again, disregarding the strict bandwidth, the older subjects started to deviate their reaches towards the right at around $204 \mathrm{~ms}$ and to the left at around $222 \mathrm{~ms}$ $\left(t_{(7)}=-2.47, P<0.05 ; t_{(7)}=2.55, P<0.05\right.$, respectively). Note that they are still initiating their deviation later than the younger sample.

For the location stop condition, what is remarkable is the inability of both age-groups to inhibit a correction deviation towards the target perturbation (see Fig. 2). Both groups were correcting towards the perturbation even if instructed not to do so. Again the same increased variability of the unperturbed trials bandwidth is observed for the older 
Fig. 2 Mean 2D vector (deg) per time slice (ms) for the younger (a) and older (b) groups per final target position (left, right and non-perturbed trials) for the location stop condition. The traced line represents mean values of unperturbed trials with \pm 2 standard deviations
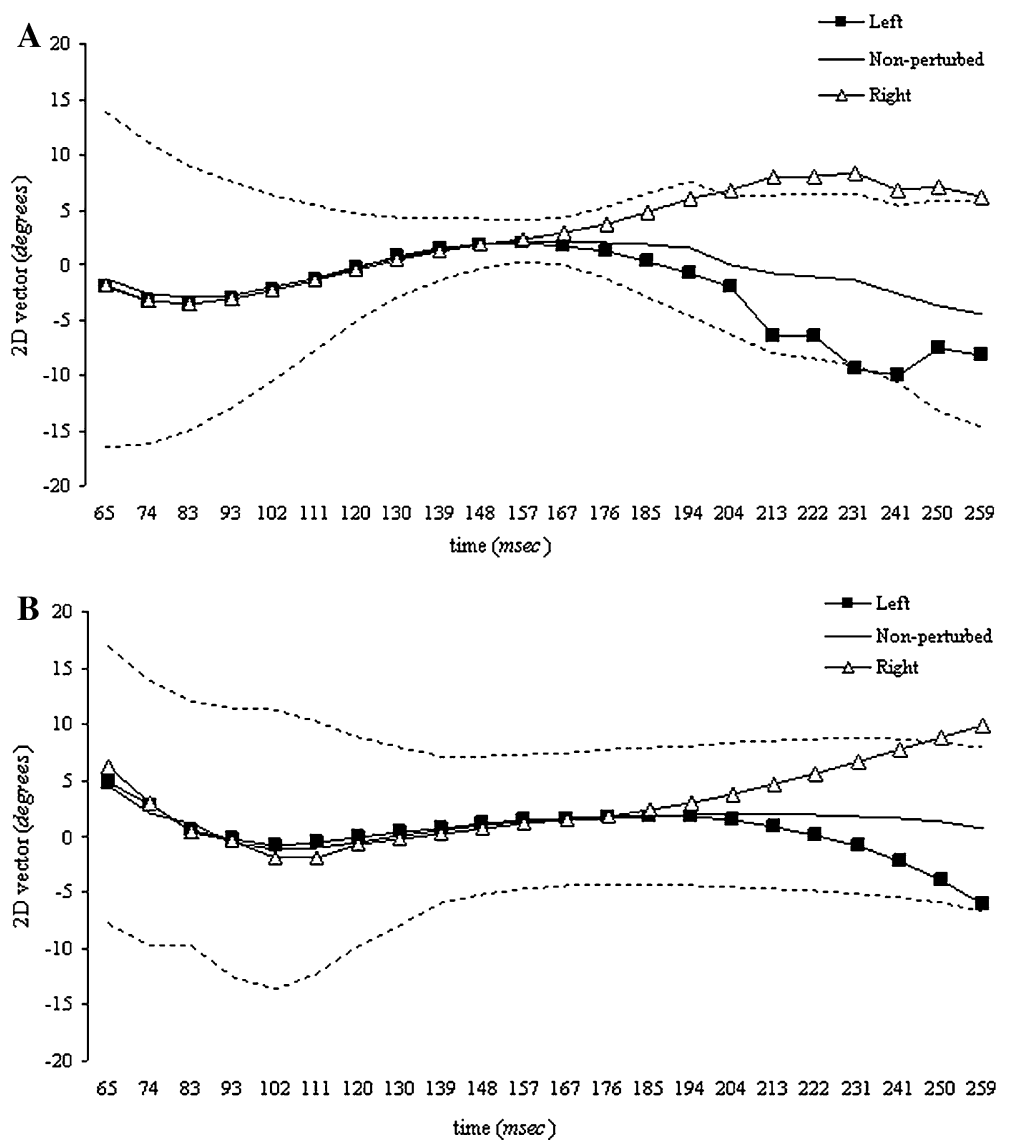

sample (Fig. 2b), when compared to the younger group (Fig. 2a). In addition, note that the $2 \mathrm{D}$ vector values were outside the bandwidth on both groups for the right perturbation, but only marginally outside for the leftward perturbation. This last result confirms the effect of side obtained regarding the proportion of disallowed corrections in the stop condition that is subjects made more disallowed corrections when the target was perturbed rightwards than leftwards. Finally, it is noteworthy that similar correction initiation timings are observed between the two conditions, reflecting a consistency regarding the timing of both successful corrections (go condition) and disallowed corrections (stop condition).

In sum, it seems that the only age-related differences we found were that the older adults were more variable and initiated both their corrections and stops later in time. However, this slowness and variability of the older group appears not to be specific to online corrective movements or inhibitory movements as the same pattern was observed for unperturbed trials. Even the 2D vector trajectories were very similar between the groups, the only difference being the timing and variability of the movement.

Therefore, the spatial trajectory analysis confirms the earlier findings with older subjects taking longer to initiate, execute, correct and inhibit a pointing movement, but achieving the same final accuracy as the younger group. Finally, with respect to the side to which the target perturbation occurred, it was found that all participants took longer to initiate, execute and interrupt a corrective pointing movement when the target was perturbed in a leftward compared to a rightward direction. Interestingly, we also found that all subjects had more difficulty inhibiting a corrective movement in response to a right target displacement, when compared to a left one.

\section{Discussion}

In agreement with the findings of Sarlegna (2006) and Plotnick et al. (1998), older subjects were markedly slower in initiating a correction towards target perturbations. However, they were as accurate as young subjects, their trajectories were smooth and they occluded the target perfectly. This finding suggests that although older subjects have longer latencies to initiate an adjustment of an ongoing movement, they can achieve it with the same accuracy as young controls.

Moreover, this slowness was not specific to perturbed targets, as the older group was significantly slower and more variable even when executing goal-directed movements 
to stationary targets (albeit as accurate). Consistent with previous findings, our data indicates that older adults take longer to initiate, execute and correct and are also more variable in their reaches (Haaland et al. 1993; Yan 2000). Whether these 'deficits' are related to neuromuscular changes (e.g. Booth et al. 1994), a decrease in processing speed (e.g. Salthouse 2000; Verhaeghen and Cerella 2002, for a review), a deterioration of the ability to generate a movement plan (e.g. Pratt et al. 1994; Yan et al. 1998) or a dysregulation of motor generation processes (Yordanova et al. 2004) is still not clear.

It might be argued that our classification of a successful correction did not strictly fit criteria of an 'automatic' fast correction, as the timing of the correction initiations was noticeably larger than the one reported previously (e.g. Prablanc and Martin 1992) but differences in methodology have to be taken into account (e.g. target distance and trial classifications based on strict individual 95\% confidence intervals obtained from unperturbed trials). Indeed, the 2D reaching trajectories without the strict bandwidth criteria showed correction timing comparable to that of previous studies.

Moreover, the time it took participants to initiate their correction towards the perturbation was longer than the time taken to update their movements following the perturbation. In particular, while the correction was initiated at approximately $279 \mathrm{~ms}$, it was completed in only $226 \mathrm{~ms}$. This finding suggests that the second submovement was executed more rapidly than the first. Also, the total movement time of the perturbed trials was generally increased by $50 \mathrm{~ms}$ only, compared to the movements made to the stationary targets. In agreement with previous work (e.g. Paulignan et al. 1991), our data suggests that these corrections did not require the re-programming of a new motor output; if they did participants would have taken more time to execute the correction.

Finally, the time it took subjects to stop their ongoing reach was longer than the time with which they initiated a correction. Based on the idea that stop responses require awareness and intentional reprogramming (Cressman et al. 2006; Pisella et al. 2000), we would state that our correction times were fast enough to escape intentional motor control. In line with this argument is the observation that only $56 \%$ of the corrections could be inhibited by an instruction to stop the movement: even when the target perturbation should have led to a 'pullback' response, a spontaneous correction still occurred. The findings confirm previous claims that online corrections to target location changes can have such automaticity that they escape intentional motor control (Pisella et al. 2000; Day and Lyon 2000).

We also analysed the influence of aging in inhibiting a fast ongoing motor response and found that, despite the increase in the timing measures and the greater variability, older adults were as able as young controls to stop their ongoing reach in response to a target location change. This outcome is surprising in view of the proposal that executive cognitive functions supported by frontal lobe structures show the strongest declines with aging (Phillips and Della Sala 1998). In fact, alterations in frontal lobe morphology and metabolism have been frequently reported in older subjects (for review see West 1996). In addition, it has been proposed that aging is accompanied with a decrease of inhibitory functions and that additional brain areas are activated for the execution of these tasks in older subjects (e.g. Nielson et al. 2002). Other authors have proposed that as the prefrontal cortex plays a crucial role in inhibition-type tasks, aging is accompanied by specific reduced activity in these areas (e.g. Chao and Knight 1997) and indeed West (1996) proposed that the prefrontal cortex plays a crucial role in aging.

In the motor domain, it has been previously shown that with aging comes an increased difficulty to inhibit a primed motor response (Potter and Grealy 2006). We did not find this pattern in our paradigm, as there was no decrease in the accuracy of the inhibitory motor control with aging, although there was a general slowing and increased variability in both online and inhibitory movements to both perturbed and unperturbed targets. Our data does agree with previous findings on oculomotor inhibition where aging did not result in increased capture effects (Eenshuistra et al. 2004; Colcombe et al. 2003; Kramer et al. 1999). Interestingly, Kramer et al. (2000) found that both elderly and young participants misdirected their saccades in an equivalent proportion of trials when awareness of the distracter was low, but when the distracter was brighter than the search stimuli older subjects were less efficient in inhibiting saccades towards these distracters. We would thus agree with Kramer et al. (1994) that different processes are involved in the different forms of inhibition and aging might not be uniquely implicated in all of these.

For all subjects reaction times were longer for the location stop than the location go condition. This finding might be related to task difficulty as a motor inhibition may require greater preparation time. In addition, it seemed that the difference in reaction times between younger and older participants was qualitatively (if not significantly) higher in the stop condition, which is in agreement with previous findings of increased latencies for older subjects regarding more complex tasks (Kok et al. 2000; Salthouse 2000; Yordanova et al. 2004).

Finally, regarding the effects of perturbation side, we found that both groups were faster when correcting to a right rather than a left target displacement. Similar laterality effects were observed for stop time, in that all subjects took longer to stop to the left than to the right side of space. In 
addition, both groups were less efficient to inhibit a correction to a right compared to a left target perturbation. The higher speed of the right hand in right hemispace could be due to biomechanical effects (Carey et al. 1996). Future studies should analyse this asymmetry in greater depths asking subjects to reach with the left hand also, to both sides of space.

Finally, the greater speed of the right hand in right hemispace may be related to the increased variable error obtained for the right target displacement: as subjects were faster when moving to this side, the endpoints of their movements might have become more variable.

To conclude, our findings show that aging does not specifically influence the ability to perform or inhibit fast online corrections to target location changes. Further studies of motor control across the lifespan are needed as a general slowing is not necessarily indicative of poor performance, although a correlation between risk of mortality and change in strength and motor performance (Buchamn et al. 2007) has been found. In particular, just exactly what type of inhibitory functions are implicated in age-related cognitive decline, has to be further elaborated.

Acknowledgments We are very grateful to Robert McIntosh for the stimulus presentation and analysis programmes. We also thank Stephen H Butler for technical support at the setup stage and David Carey and Thomas Schenk for most constructive comments on an earlier version of this manuscript. This work was funded by a grant (SFRH/BD/23230/2005) from the Foundation for Science and Technology (FCT, Portugal) to S. Rossit.

\section{References}

Annett M (1967) The binomial distribution of right, mixed and left handedness. Q J Exp Psychol 19:327-333

Aron AR, Poldrack RA (2006) Cortical and subcortical contributions to stop signal response inhibition: role of the subthalamic nucleus. J Neurosci 26:2424-2433

Booth FW, Weeden SH, Tseng BS (1994) Effect of aging on human skeletal muscle and motor function. Med Sci Sport Exerc 26:556-560

Buchamn AS, Wilson RS, Boyle PA, Bienias J, Bennett D (2007) Change in motor function and risk of mortality in older persons. $\mathrm{J}$ Am Geriatr Soc 55:11-19

Calautti C, Serrati C, Baron J-C (2001) Effects of age on brain activation during auditory-cued thumb-to-index opposition: a positron emission tomography study. Stroke 32:139-146

Carey D, Hargreaves EL, Goodale MA (1996) Reaching to ipsilateral or contralateral targets: within-hemisphere visuomotor processing cannot explain hemispatial differences in motor control. Exp Brain Res 112:496-504

Castiello U, Paulignan Y, Jeannerod M (1991) Temporal dissociation of motor responses and subjective awareness: a study in normal subjects. Brain 114:2639-2655

Chao LL, Knight RT (1997) Prefrontal deficits in attention and inhibitory motor control with aging. Cereb Cortex 7:63-69

Chua R, Enns JT (2005) What the hand can't tell the eye: illusion of space constancy during accurate pointing. Exp Brain Res 162:109-114
Colcombe AM, Kramer AF, Irwin D E, Peterson MS, Colcombe S, Hahn S (2003) Age-related effects of attentional and oculomotor capture by onsets and colour singletons as a function of experience. Acta Psychol 113:205-225

Curtis CE, Cole MW, Rao VY, D’Esposito M (2004) Cancelling planned action: an fMRI study of countermanding saccades. Cereb Cortex 15:1281-1289

Cressman EK, Franks IM, Enns JT, Chua R (2006) No automatic pilot for visually guided aiming based on colour. Exp Brain Res 171:174-183

Day BL, Lyon IN (2000) Voluntary modification of automatic arm movements evoked by motion of a visual target. Exp Brain Res 130:159-168

Eenshuistra RM, Ridderinkhof KR, van der Molen MW (2004) Agerelated changes in antisaccade task performance: inhibitory control or working-memory engagement? Brain Cogn 56:177-188

Fecteau JH, Chua R, Franks I, Enns JT (2001) Visual awareness and on-line modification of action. Can J Exp Psychol 55:106-112

Goodale MA, Milner AD (2005) Sight unseen: an exploration of conscious and unconscious vision. Oxford University Press, Oxford

Goodale MA, Pélisson D, Prablanc C (1986) Large adjustments in visually guided reaching do not depend on vision of the hand or perception of target displacement. Nature 320:748-750

Gréa H, Pisella L, Rossetti Y, Desmurget M, Tilikete C, Grafton S, Prablanc C, Vighetto A (2002) A lesion of the posterior parietal cortex disrupts on-line adjustments during aiming movements. Neuropsychologia 40:2471-2480

Haaland KY, Harrington DL, Grice JW (1993) Effects of aging in planning and implementing arm movements. Psychol Aging 8:617632

Heuninckx S, Wenderoth N, Debaere F, Peeters R, Swinnen SP (2005) Neural basis of aging: the penetration of cognition into action control. J Neurosci 25:6787-6796

Hutchinson S, Kobayashi M, Horkan CM, Pascual-Leone A, Alexander MP, Schlaug G (2002) Age-related differences in movement representation. Neuroimage 17:1720-1728

Johnson H, Van Beers RJ, Haggard P (2002) Action and awareness in pointing tasks. Exp Brain Res 146:451-459

Kok A (2000) Age-related changes in involuntary and voluntary attention as reflected in components of the event-related potential (ERP). Biol Psychol 54:107-143

Kramer AF, Hahn S, Irwin DE, Theeuwes J (1999) Attentional capture and aging: implications for visual search performance and oculomotor control. Psychol Aging 14:135-154

Kramer AF, Humphrey D, Larish J, Logan G, Strayer D (1994) Aging and inhibition: beyond a unitary view of inhibitory processing in attention. Psychol Aging 9:491-512

Kramer AF, Hahn S, Irwin DE, Theeuwes J (2000) Age differences in the control of looking behaviour: do you know where the eyes have been? Psychol Sci 11:210-217

Lincort AE, Folk CL, Hoyer WJ (1997) Effects of aging on voluntary and involuntary shifts of attention. Aging Neuropsychol Cogn 4:290-303

Mattay VS, Fera F, Tessitore A, Hariri AR, Das S, Callicott JH, Weinderger DR (2002) Neurophysiological correlates of age-related changes in motor function. Neurology 58:630-635

Mirabella G, Pani P, Paré M, Ferraina S (2006) Inhibitory control of reaching movements in humans. Exp Brain Res 174:240-255

Nielson KA, Langenecker SA, Garavan H (2002) Differences in the functional neuroanatomy of inhibitory control across the life span. Psychol Aging 17:56-71

Olincy A, Ross RG, Young DA, Freedman R (1997) Age diminishes performance on an antisaccade eye movement task. Neurobio Aging 18:483-489

Paulignan Y, MacKenzie C, Marteniuk R, Jeannerod M (1991) Selective perturbation of visual input during prehension movements. I. 
The effects of changing object position. Exp Brain Res 83:502512

Pélisson D, Prablanc C, Goodale MA, Jeannerod M (1986) Visual control of reaching movements without vision of the limb. II. Evidence for fast unconscious processes correcting the trajectory of the hand to the final position of a double-step stimulus. Exp Brain Res 62:303-311

Phillips LH, Della Sala S (1998) Aging, intelligence and anatomical segregation in the frontal lobes. Learn Individ Differ 10:217-243

Pisella L, Gréa H, Tilikete C, Vighetto A, Desmurget M, Rode G, Boisson D, Rossetti Y (2000) An 'automatic pilot' for the hand in human posterior parietal cortex: toward reinterpreting optic ataxia. Nature 3:729-735

Plotnik M, Flash T, Inzelberg R, Schechtman, Korczyn AD (1998) Motor switching abilities in Parkinson's disease and old age: temporal aspects. J Neurol Neurosurg Psychiatry 65:328-337

Potter LM, Grealy MA (2006) Aging and inhibitory error on a motor shift of set task. Exp Brain Res 171:56-66

Potter LM, Grealy MA (2007) Aging and inhibition of a prepotent motor response during an ongoing action. Aging Neuropsychol Cogn (in press)

Prablanc C, Martin O (1992) Automatic control during hand reaching at undetected two-dimensional target displacements. J Neurophysiol 67:455-469

Pratt J, Bellomo CN (1999) Attentional capture in younger and older adults. Aging Neuropsychol Cogn 6:19-31

Pratt J, Chasteen AL, Abrams RA (1994) Rapid aimed limb movements: age differences and practise effects in component submovements. Psychol Aging 9:325-334

Salthouse TA (1979) Adult age and the speed/accuracy tradeoff. Ergonomics 22:811-821

Salthouse TA (2000) Aging and measures of processing speed. Biol Psychol 54:35-54
Sarlegna FR (2006) Impairment of online control of reaching movements with aging: a double-step study. Neurosci Lett 403:309314

Schall JD (2001) Neural basis of deciding, choosing and acting. Nat Neurosci 2:33-42

Schall JD, Hanes DP, Taylor TL (2000) Neural control of behavior: countermanding eye movements. Psychol Res 63:299-307

Schall JD, Stuphron V, Brown JW (2002) Monitoring and control of action by the frontal lobes. Neuron 36:309-322

Verhaeghen P, Cerella J (2002) Aging, executive control, and attention: a review of meta-analysis. Neurosci Biobehav Rev 26:849857

Walker N, Philbin DA, Fisk AD (1997) Age-related differences in movement control: adjusting submovement structure to optimize performance. J Gerontol Psychol Sci 52:40-52

Ward NS, Frackowiak RSJ (2003) Age-related changes in the neural correlates of motor performance. Brain 126:873-888

Welford AT, Norris AH, Shock NW (1969) Speed and accuracy of movement and their changes with age. Acta Psychol 30:3-15

West R (1996) An application of prefrontal cortex function theory to cognitive aging. Psychol Bull 120:272-292

Wu T, Hallett M (2005) The influence of normal ageing on automatic movements. J Physiol 562:605-615

Yan JH (2000) Effects of aging in linear and curvilinear arm movements. Exp Aging Res 26:393-407

Yan JH, Thomas JR, Stelmach GE (1998) Aging and rapid aiming arm movement control. Exp Aging Res 24:155-168

Yordanova J, Kolev V, Hohnsbein J, Falkenstein M (2004) Sensorimotor slowing with aging is mediated by a functional dysregulation of motor-generation processes: evidence from high-resolution event-related potentials. Brain 127:351-362 